\title{
EFEITO DO PH NA ADSORÇÃO DE METAIS DE UMA SOLUÇÃO SINTÉTICA UTILIZANDO RESINA QUELANTE DOWEX XUS43605*
}

\section{Resumo}

Isadora Dias Perez ${ }^{1}$ Mónica Maria Jiménez Correa² Flávia Paulucci Cianga Silvas ${ }^{3}$ Jorge Alberto Soares Tenório ${ }^{4}$ Em vista da crescente geração de efluentes contendo metais, busca-se por técnicas sustentáveis capazes de recuperá-los, devido à toxicidade e ao valor econômico agregado. Os metais ainda contribuem com a poluição dos recursos hídricos. Desta maneira, o presente trabalho investiga a adsorção dos metais presentes num efluente produzido na mineração (licor) do níquel, através da resina quelante de troca iônica Dowex XUS43605. Os experimentos foram realizados em bateladas, variando $\mathrm{pH}$, para verificar a captação pela resina do alumínio, cobalto, cromo, cobre, ferro (III), magnésio, manganês, níquel e zinco presentes no licor. A concentração dos metais na solução foi obtida pela técnica de espectrometria de fluorescência de raios-x por energia dispersiva (EDX). Os resultados mostram a capacidade de remoção dos metais pela resina, destacando $83 \%$ de adsorção do cobre e $7 \%$ do níquel, considerando $2,0 \mathrm{pH}$ e tempo de contato $2 \mathrm{~h}$. Portanto, o estudo demonstra que a resina de troca iônica pode ser utilizada para tratar efluentes, em especial, de mineração.

Palavras-chave: Troca iônica; Dowex XUS43605; Hidrometalurgia; Recuperação de metais.

\section{EFFECT OF pH ON METALS ADSORPTION OF SYNTHETIC SOLUTION USING CHELATING RESIN DOWEX XUS43605}

\section{Abstract}

In view of rising generation of wastewater containing metals, sustainable techniques to recover them are being researched targeting their toxicity and economic value. The metals also contribute to the pollution of water resources. Thus, this study investigates the adsorption of metals present in mining effluent (liquor) of nickel by chelating ion exchange resin Dowex XUS43605. The experiments were performed in batch test, varying $\mathrm{pH}$, to verify the resin uptake of aluminum, cobalt, chromium, copper, iron (III), magnesium, manganese, nickel and zinc present in the liquor. The concentration of metal in solution was obtained by the technique of $\mathrm{x}$-ray fluorescence spectrometry energy dispersive (EDX). The results show the adsorption of metals by the resin, highlighting the adsorption of $83 \%$ copper and $7 \%$ of nickel, $2.0 \mathrm{pH}$ and contact time $2 \mathrm{~h}$. Therefore, the study shows that the ion exchange resin can be used to treat effluents in particular mining.

Keywords: Ion Exchange; Dowex XUS43605; Hydrometallurgy; Recovery of metals.

1 Engenheira Ambiental, mestranda do Departamento de Engenharia Química, Escola Politécnica da Universidade de São Paulo, São Paulo, São Paulo, Brasil.

2 Engenheira Química, mestre em ciências, doutoranda do Departamento de Engenharia Química, Escola Politécnica da Universidade de São Paulo, São Paulo, São Paulo, Brasil.

3 Tecnóloga em Alimentos, doutora em engenharia química, pesquisadora no Instituto Tecnológico Vale, Ouro Preto, Minas Gerais, Brasil.

4 Engenheiro Metalurgista, doutor em engenharia metalúrgica, professor titular, Departamento de Engenharia Química, Escola Politécnica da Universidade de São Paulo, São Paulo, São Paulo, Brasil.

5 Engenheira Metalurgista, doutora em engenharia metalúrgica, professora associada, Departamento de Engenharia Química, Escola Politécnica da Universidade de São Paulo, São Paulo, São Paulo, Brasil. 


\section{INTRODUÇÃO}

A contaminação de corpos hídricos por metais tem sido alvo de preocupação ambiental. A crescente industrialização envolvendo processos, como, galvanoplastia, baterias, indústria de fertilizantes e metalurgia, tornam-se responsáveis pelo aumento da poluição das águas de lagos, rios, podendo ser ingeridos e acumulados nos seres humanos, animais e plantas [1]. Em vista disso, a recuperação de metais ganha destaque, por conta das taxas de toxicidade presentes em efluentes de mineração, bem como, a escassez desses metais como matéria-prima na natureza, por se tratar de recursos não renováveis e com valor econômico agregado [2].

A procura por técnicas e métodos sustentáveis para a recuperação dos metais cresceu ao longo dos anos, devido à expansiva produção de minérios e geração de efluentes oriundos de processos hidrometalúrgicos [3].

A lixiviação ácida sob alta pressão (HPAL) de minérios lateríticos é empregada como uma das técnicas para a recuperação de metais, como níquel e cobre a partir de minérios lateríticos. A solução produzida na lixiviação de lateritos consiste em sulfatos de metais, entre eles, $\mathrm{Al}, \mathrm{Co}, \mathrm{Cu}, \mathrm{Fe}, \mathrm{Mg}, \mathrm{Mn}, \mathrm{Ni}, \mathrm{Zn}$ obtidos através do uso de ácido sulfúrico sob pressão e em altas temperaturas variando de $245^{\circ} \mathrm{C}$ a $270^{\circ} \mathrm{C}$ [4].

A solução então segue para etapa de separação e purificação dos metais. Entre as técnicas de separação tem-se a troca-iônica, a qual é considerada simples por oferecer um desempenho eficaz e de baixo custo em relação a outros métodos, como a osmose reversa [4]. No processo de troca iônica, o efluente líquido (fase móvel) passa através de um leito estacionário (fase estacionária) formado pela resina de troca iônica [5]. A troca iônica oferece vantagens por ser menos prejudicial ao meio ambiente, se comparada a outros processos e entre estas vantagens têm-se [6]:

- o processo consome menos água e possibilita a sua recirculação no sistema;

- é viável economicamente, visto aos baixos custos operacionais e de equipamentos;

- é seletivo frente a metais de interesse proporcionando a sua separação.

Além das técnicas de separação expostas, os processos de membrana, precipitação química, osmose reversa e resina quelante também podem ser utilizados para a recuperação de metais [7]. Dessa maneira, deve-se observar as características do efluente a ser tratado, para selecionar a técnica a ser empregada.

As resinas de troca iônica variam de acordo com as características físicas e químicas, tais como, o tipo de matriz, grupo de troca e porosidade, tendo vantagens de não serem tóxicas, voláteis e evitarem a perda de solventes [8][9]. Entretanto, a escolha da resina a ser utilizada visa, principalmente, a viabilidade econômica e 0 desempenho perante os processos de sorção.

Assim, para a adsorção dos metais de interesse (Co, Cu e Ni), optou-se por resinas quelantes de troca-iônica, por adsorverem íons de metais através da combinação de interações iônicas e de coordenação, em vez de simples interações eletrostáticas de trocas catiônicas ou aniônicas [10][11]. Logo, será utilizado a resina quelante Dowex XUS43605 pela sua capacidade de adsorção e seletividade com metais de transição, bem como durabilidade [7].

\subsection{Resina Dowex XUS43605}

A resina Dowex XUS43605 possui a hidroxipropil picolilamina (HPPA) (Figura 1) como grupo funcional. A sua matriz está ligada por uma estrutura polimérica de estireno reticulado com divinilbenzeno (DVB) [12]. 


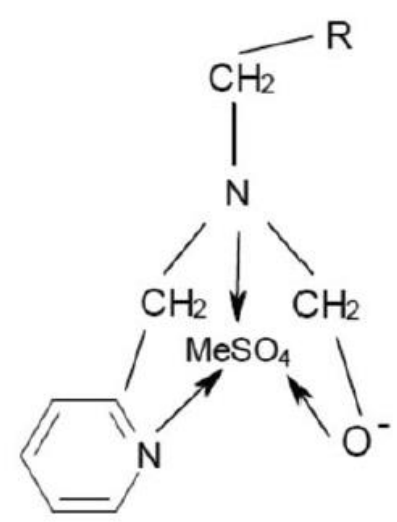

Figura 1. Estrutura química da resina.

O grupo funcional HPPA possui afinidade para metais de transição, conforme a ordem: $\mathrm{Cu}^{2+}>>\mathrm{Ni}^{2+}>\mathrm{Fe}^{3+}>\mathrm{Zn}^{2+}>\mathrm{Co}^{2+}>\mathrm{Cd}^{2+}>\mathrm{Fe}^{2+}$ [13] em pH $<2$. Além disso, a resina quelante Dowex XUS43605 possui potencial de adsorver os íons de cobre, considerando a variação de pH, conforme ilustrado na Figura 2 [13].

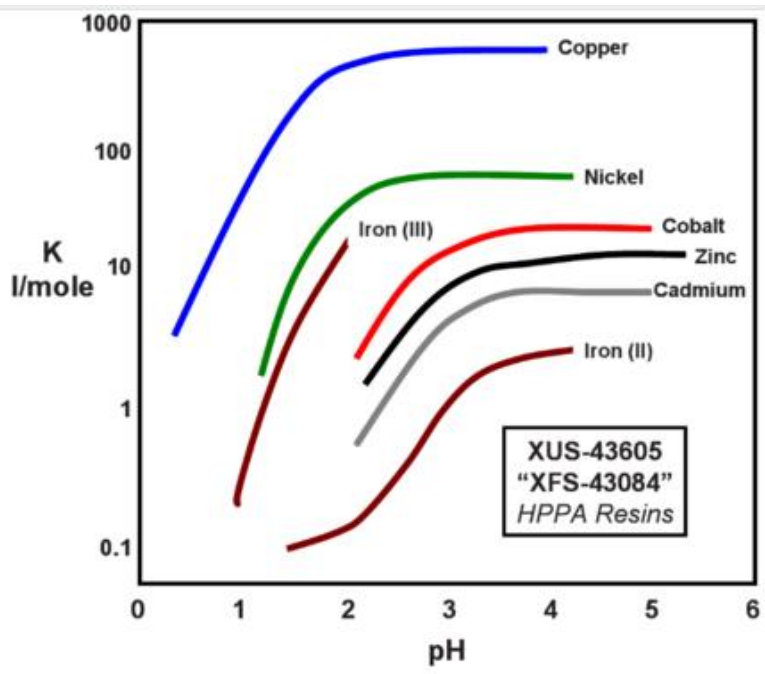

Figura 2. Seletividade da resina Dowex XUS43605 em função do pH.

As resinas do tipo HPPA, como a Dowex XUS43605, foram desenvolvidas com a intenção de substituir os extratantes orgânicos (solventes organicos) usados na mineração do cobre. Além disso, era necessário obter resinas capazes de substituir ou melhorar a remoção seletiva do níquel sobre o cobalto e eliminar a eluição por amônia alcalina, visto que resinas como bis-picolilamina (BPA) e ácido iminodiacético (HIDA) apresentam alta seletividade com o cobre havendo dificuldade de elui-lo e impossibilitando que a eluição seja feita com ácido sulfúrico, como acontece com resinas HPPA [12]. Por consequência, o presente trabalho pretende avaliar o efeito do $\mathrm{pH}$ na adsorção dos metais presentes num licor produzido na mineração de níquel usando a resina quelante Dowex XUS43605. 


\section{MATERIAIS E MÉTODOS}

\subsection{Materiais}

A resina adotada para este trabalho foi a resina quelante Dowex XUS43605. A Tabela 1 apresenta as características da resina.

Tabela 1. Características físicas e químicas da resina comercial Dowex XUS43605[14]

\section{Fabricante}

Grupo Funcional

Forma lônica

Umidade

Tamanho

Capacidade de troca

\section{Dow Chemical Company}

HPPA

Macroporosa St-DB

Ácido Sulfúrico

$40-60 \%$

$320 \mu \mathrm{m}$

$35 \mathrm{~g} / \mathrm{L}$ de $\mathrm{Cu}$

\subsection{Pré-tratamento da resina}

$\mathrm{Na}$ etapa de pré-tratamento, a resina foi lavada com $\mathrm{HCl} 4 \mathrm{M}$ e $\mathrm{NaOH} 2 \mathrm{M}$, a seguir adicionou-se água deionizada, a fim de remover todas as impurezas. O procedimento foi desenvolvido com o auxílio de frascos agitados de $250 \mathrm{~mL}$ e shaker refrigerado de bancada com velocidade de 200rpm. Para obter purificação, a resina foi lavada com água destilada a $60^{\circ} \mathrm{C}$ e em seguida com etanol absoluto. Finalmente a resina foi seca em estufa $\left(60^{\circ} \mathrm{C}\right)$ por aproximadamente $2 \mathrm{~h}$.

\subsection{Preparo do licor sintético}

O licor sintético utilizado neste trabalho foi preparado usando os seguintes reagentes de grau analítico $\mathrm{Al}_{2}\left(\mathrm{SO}_{4}\right)_{3 .} 17 \mathrm{H}_{2} \mathrm{O}, \mathrm{CoSO}_{4} .7 \mathrm{H}_{2} \mathrm{O}, \mathrm{Cr}_{2}\left(\mathrm{SO}_{4}\right)_{3} .8 \mathrm{H}_{2} \mathrm{O}, \mathrm{CuSO}_{4} .5 \mathrm{H}_{2} \mathrm{O}$, $\mathrm{Fe}_{2}\left(\mathrm{SO}_{4}\right)_{3} .8 \mathrm{H}_{2} \mathrm{O}, \mathrm{MgSO}_{4} .7 \mathrm{H}_{2} \mathrm{O}, \mathrm{MnSO}_{4} . \mathrm{H}_{2} \mathrm{O}, \mathrm{NiSO}_{4} .6 \mathrm{H}_{2} \mathrm{O}, \mathrm{ZnSO}_{4} .7 \mathrm{H}_{2} \mathrm{O}$, os quais foram diluídos com água deionizada $A$ concentração de cada metal contido no licor está listada na Tabela 2.

Tabela 2. Composição do licor sintético

\begin{tabular}{ll} 
Metal & Concentração $(\mathbf{p p m})$ \\
\hline $\mathrm{Al}$ & 4101,54 \\
\hline $\mathrm{Co}$ & 78,06 \\
\hline $\mathrm{Cr}$ & 195,2 \\
$\mathrm{Cu}$ & 146,91 \\
\hline $\mathrm{Fe}$ & $18.713,51$ \\
\hline $\mathrm{Mg}$ & $7.774,54$ \\
\hline $\mathrm{Mn}$ & 397,17 \\
\hline $\mathrm{Ni}$ & $2.434,21$ \\
\hline $\mathrm{Zn}$ & 36,67
\end{tabular}

\subsection{Ensaios em batelada}

Os experimentos foram realizados a $25^{\circ} \mathrm{C}$ em frascos agitados de $250 \mathrm{~mL}$, usando-se $1 \mathrm{~g}$ de resina e $50 \mathrm{~mL}$ de solução. A agitação foi feita em um shaker de bancada mantendo velocidade de 200rpm. O parâmetro analisado foi o pH. Com a intenção de 
determinar o melhor $\mathrm{pH}$ para a adsorção dos metais, os ensaios foram realizados variando o $\mathrm{pH}$ em uma faixa de $0,5-2,0 \mathrm{com}$ um tempo de contato de $2 \mathrm{~h}$. Para ajustar o $\mathrm{pH}$ para o valor desejado utilizou-se $\mathrm{H}_{2} \mathrm{SO}_{4} 5 \mathrm{M}$ e/ou $\mathrm{NaOH} 2 \mathrm{M}$. Para identificar e quantificar os íons metálicos presente na solução, o licor sintético foi submetido à análise química através da técnica de espectrometria de fluorescência de raios-x de energia dispersiva (EDX), em um equipamento da marca PANalytical modelo Epsilon $3 \mathrm{XL}$. Os resultados encontrados foram utilizados como valores de referência para 0 cálculo da capacidade de adsorção (Equação 1).

$$
q_{e}=\frac{\left(C_{o}-C_{e}\right)}{M} x V
$$

Onde qe é a capacidade de adsorção no equilibro $(\mathrm{mg} / \mathrm{g})$, V é volume do licor ( $\mathrm{L})$, M é a massa da resina seca usada em cada ensaio $(\mathrm{g})$ e $\mathrm{C}_{0}$ e $\mathrm{C}_{e}$ são as concentrações iniciais e finais dos íons metálicos na solução (mg/L), respectivamente. A seguir, foi calculado o coeficiente de distribuição ( $\left.K_{d}\right)$ (Equação 2).

$$
K_{d}=\frac{C_{\text {resina }}}{C_{\text {solução }}} \times \frac{V}{M}
$$

Logo, $\mathrm{K}_{d}(\mathrm{~mL} / \mathrm{g})$ é definido pelo número do íon fixado na resina $\mathrm{C}_{\text {resina }}(\mathrm{mg} / \mathrm{L})$, dividido pelo número deste íon na solução $\mathrm{C}_{\text {solução }}(\mathrm{mg} / \mathrm{L})$, multiplicado pelo volume do licor $(\mathrm{mL})$, dividido pela massa da resina seca $(\mathrm{g})$ [5].

A porcentagem adsorvida de cada metal foi obtida, conforme a Equação 3.

$$
\%=\frac{K_{d}}{K_{d}+\left(\frac{V}{M}\right)} * 100
$$

\section{RESULTADOS E DISCUSSÃO}

\subsection{Efeito do pH}

Os ensaios visando analisar o efeito do $\mathrm{pH}$ foram feitos utilizando a técnica de batelada em temperatura de $25^{\circ} \mathrm{C}$, tempo de contato de $2 \mathrm{~h}$ e variando o $\mathrm{pH}$ entre 0,5 2,0. O pH da solução é um dos parâmetros capaz de controlar e impactar o processo de adsorção da resina afetando a carga superficial do sorvente [1] [15], pois em situações em que o pH varia, o grupo quelante forma complexos ao se ligarem aos íons metálicos. Assim, estudou-se a capacidade da resina em adsorver os íons da solução (Figura 3). 


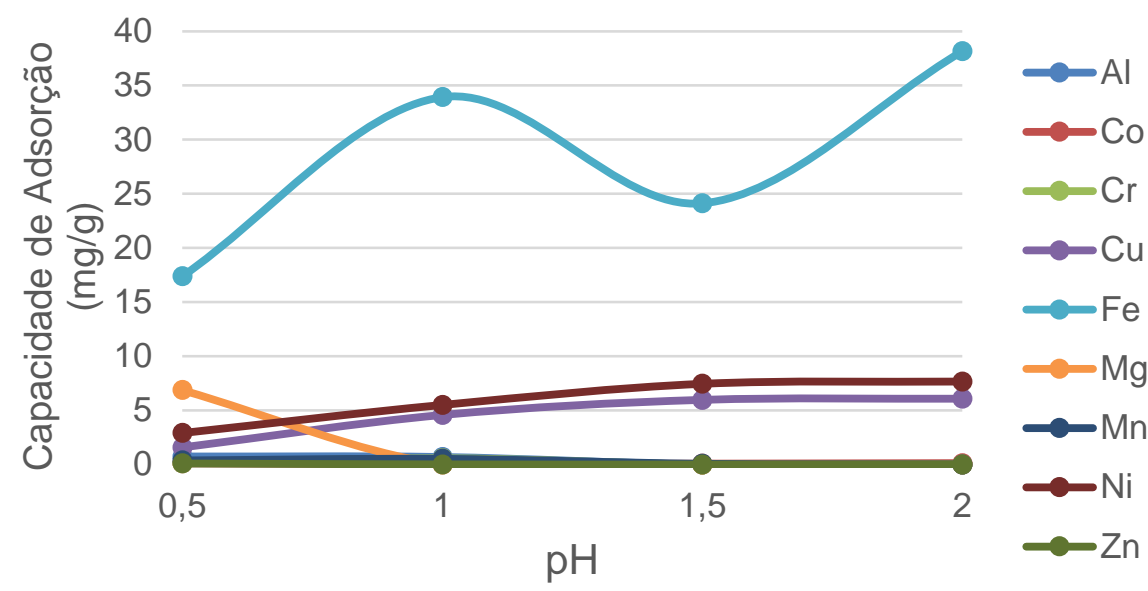

Figura 3. Efeito do pH na capacidade de adsorção dos metais da solução sintética.

$\mathrm{Na}$ Figura 3, pode ser observado que em diferentes valores de $\mathrm{pH}$, a relação entre a resina e os íons metálicos apresentou um comportamento específico, o que pode ter sido devido à alteração do grupo funcional da resina pela protonação. Tal efeito é verificado para $\mathrm{pH}<1,0$, quando o grupo quelante está protonado impedindo a adsorção dos íons metálicos, os quais foram repelidos, justificando a baixa sorção dos metais pela resina [16].

Para os valores entre 1,5 e 2,0, verificou que o equilíbrio foi atingido, tornando a capacidade de adsorção da resina estável. Já para pH 0,5, a adsorção é praticamente nula, pois ocorre a protonação completa do grupo quelante.

Dessa forma, com o aumento do $\mathrm{pH}$, a resina passará a ter caráter negativo, proporcionando atração eletrostática entre os íons metálicos que estejam carregados positivamente, favorecendo a adsorção dos íons. Logo, com a diminuição do pH, haverá competição entre os íons $\mathrm{H}^{+} \mathrm{e}$ os íons metálicos, os quais desejam fixarem à superfície do adsorvente.

Entretanto, existe também o caso, em que o pH aumenta e a adsorção diminui, isso está relacionado com a formação de complexos insolúveis composto pelos hidróxidos dos íons metálicos presentes na solução [15].

Sendo assim, a adsorção dos metais foi condicionada a solução ácida com pH máximo de 2,0, a fim de evitar a precipitação do íon férrico, a qual inviabiliza a determinação da concentração real adsorvida deste metal pela resina [17].

Já a Figura 4 expressa o resultado obtido do coeficiente de distribuição. 


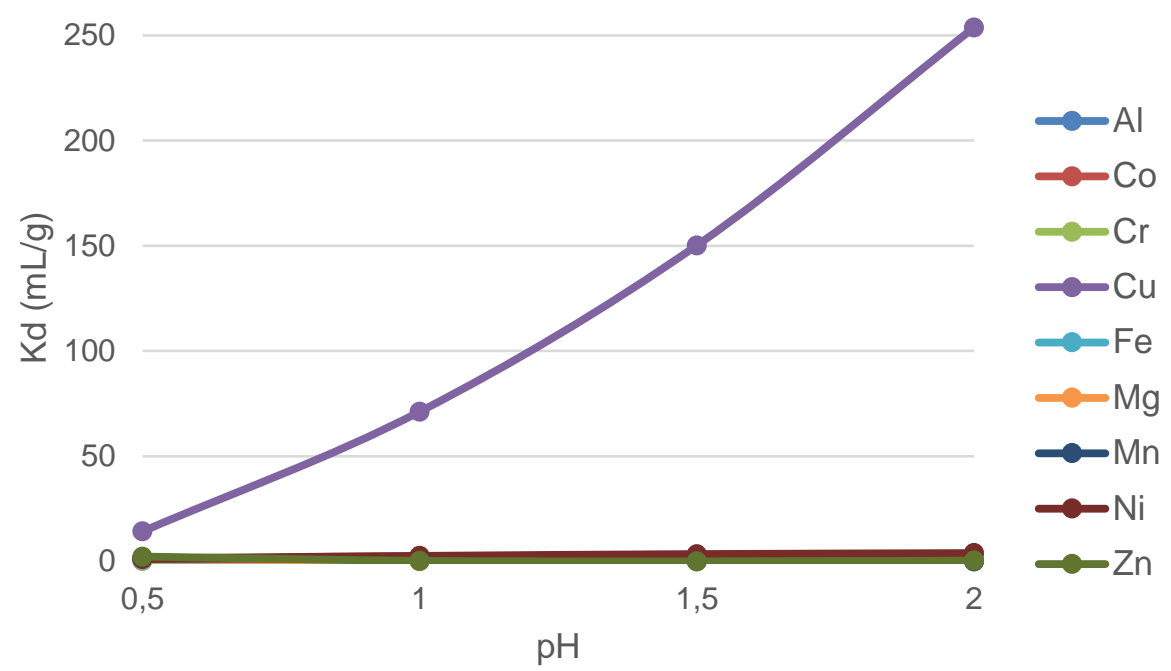

Figura 4. Coeficiente de distribuição $\left(\mathrm{K}_{\mathrm{d}}\right)$ em função do $\mathrm{pH}$

Há destaque para os valores encontrados para o $\mathrm{Cu}^{2+}$, sendo o valor mínimo $14 \mathrm{~mL} / \mathrm{g}$ e o valor máximo $253 \mathrm{~mL} / \mathrm{g}$. Enquanto isso, os valores para o $\mathrm{Ni}^{2+}$ compreenderam o intervalo de 1,4-4,0 mL/g. Já o $\mathrm{Fe}^{3+}$ obteve variação de $1,0-2,2 \mathrm{~mL} / \mathrm{g}$. Dessa forma, é possível aferir que o $\mathrm{Cu}^{2+}, \mathrm{Ni}^{2+}$ e $\mathrm{Fe}^{3+}$ tiveram comportamentos diferentes, assim como os demais metais contidos na solução sintética.

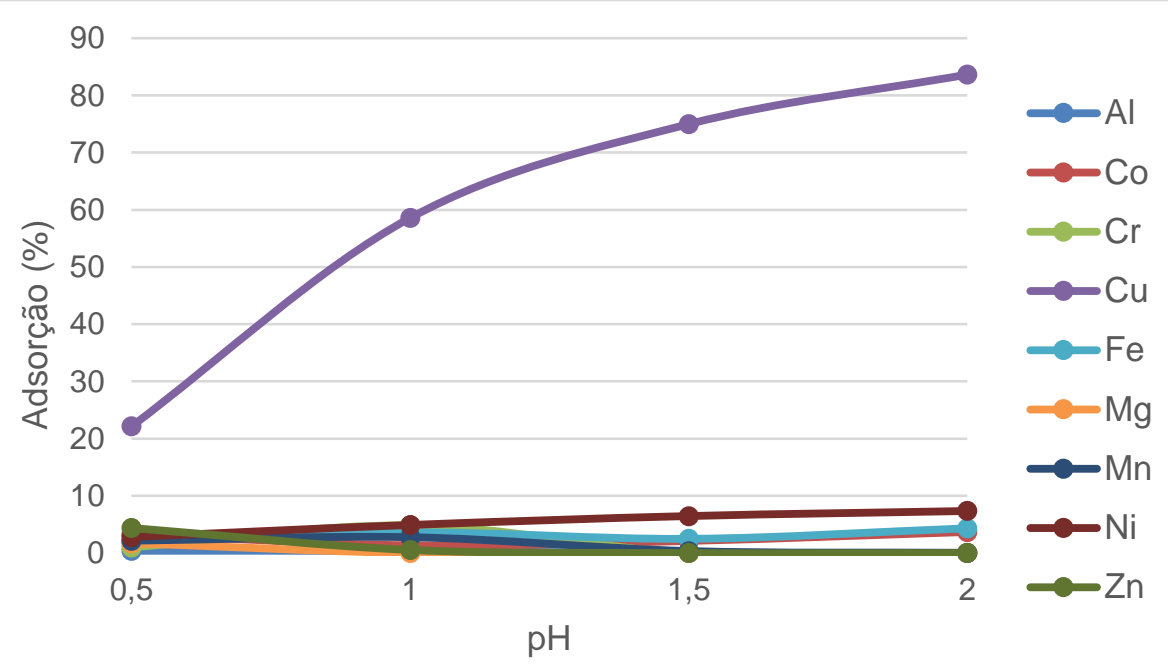

Figura 5. Porcentagem adsorvida dos metais pela resina quelante Dowex XUS43605.

Conforme a Figura 3 e 5, a resina apresentou grande afinidade com o $\mathrm{Cu}^{2+}$ e Ni${ }^{2+}$, em relação ao $\mathrm{Co}^{2+}, \mathrm{Cr}^{2+}, \mathrm{Mg}^{2+}, \mathrm{Mn}^{2+}$ e $\mathrm{Zn}^{2+}$. A máxima adsorção do $\mathrm{Cu}^{2+}$ e do e $\mathrm{Ni}^{2+}$ foi em $\mathrm{pH}=2$, com capacidade de adsorção de $6,08 \mathrm{mg} / \mathrm{g} \mathrm{e} 7,70 \mathrm{mg} / \mathrm{g}$, respectivamente. Isso significa que em $\mathrm{pH}=2,0,83 \%$ de $\mathrm{Cu}^{2+}$ foi adsorvido pela resina, enquanto que para o $\mathrm{Ni}^{2+}$, apenas $7 \%$.

Já no caso do $\mathrm{Fe}^{3+}$, a adsorção foi a maior entre os íons metálicos estudados, sendo

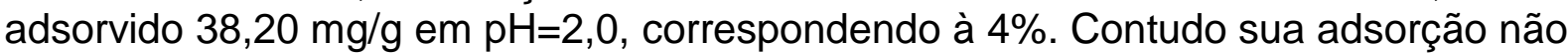
é justificada pela afinidade com a resina, mas pela sua alta concentração frente aos outros metais da solução sintética, uma vez que sua concentração ultrapassa 18000 ppm, enquanto a concentração de outros metais como o $\mathrm{Cu}^{2+}$ é de aproximadamente $150 \mathrm{ppm}$. 
Dessa forma, a seletividade da resina não seguiu a ordem prevista: $\mathrm{Cu}^{2+}>>\mathrm{Ni}^{2+}>$ $\mathrm{Fe}^{3+}>\mathrm{Zn}^{2+}>\mathrm{Co}^{2+}>\mathrm{Cd}^{2+}>\mathrm{Fe}^{2+}$, refletindo na interação entre os íons metálicos da solução com o grupo funcional da resina.

\section{CONCLUSÃO}

O presente trabalho estudou o uso da resina quelante Dowex XUS43605 para a recuperação de metais contidos em um licor sulfúrico produzido na mineração do níquel. A investigação avaliou o efeito da variação do $\mathrm{pH}$ na adsorção dos íons pela resina.

Desta forma, os experimentos mostraram que a resina Dowex XUS43605 adsorveu $6,08 \mathrm{mg} / \mathrm{g}$ de $\mathrm{Cu}^{2+}$ e $7,70 \mathrm{mg} / \mathrm{g}$ de $\mathrm{Ni}^{2+}$, sendo que a máxima adsorção ocorreu em $\mathrm{pH}=2$, considerando tempo de contato $2 \mathrm{~h}$.

No caso do $\mathrm{Fe}^{3+}$, a adsorção foi a maior entre os íons metálicos estudados, sendo adsorvido $38,20 \mathrm{mg} / \mathrm{g}$ em $\mathrm{pH}=2,0$. Contudo sua adsorção não é justificada pela afinidade com a resina, mas pela sua alta concentração em relação aos outros metais da solução sintética.

\section{Agradecimentos}

À Coordenação de Aperfeiçoamento de Pessoal de Nível Superior (CAPES) pelo apoio financeiro à bolsa de mestrado.

Ao Conselho Nacional de Desenvolvimento Científico e Tecnológico (CNPq) pelo suporte financeiro à bolsa de doutorado.

À Fundação de Amparo à Pesquisa do Estado de São Paulo (FAPESP) pelo auxílio financeiro através do projeto de pesquisa 2012/51871-9.

À Vale S.A. pelo apoio financeiro.

\section{REFERÊNCIAS}

1. Kumar R, Kumar M, Ahmad R, Barakat MA. L-Methionine modified Dowex-50 ionexchanger of reduced size for the separation and removal of $\mathrm{Cu}(\mathrm{II})$ and $\mathrm{Ni}(\mathrm{II})$ from aqueous solution. Chemical Eng J. 2013;218:32-8.

2. Lin Z, Zhang Y, Chen Y, Qian H. Extraction and recycling utilization of metal ions (Cu2+, $\mathrm{Co} 2+$ and $\mathrm{Ni2}+$ ) with magnetic polymer beads. Chem Eng J. Elsevier B.V.; 2012;200-202:104-12.

3. Diniz C V., Ciminelli VST, Doyle FM. The use of the chelating resin Dowex M-4195 in the adsorption of selected heavy metal ions from manganese solutions. Hydrometallurgy. 2005;78(3-4):147-55.

4. Mendes FD, Martins AH. Selective nickel and cobalt uptake from pressure sulfuric acid leach solutions using column resin sorption. Int J Miner Process. 2005;77(1):53-63.

5. $\quad$ Abrão A. Operações de Troca lônica. São Paulo; 2014. 201 p.

6. Zontov N. Potential benefits of resin-in-pulp for PAL plants. Perth, Western Australia: Proceedings ALTA 2001 Nickel/Cobalt-7; 2001. p. 13.

7. El-Bahy SM, El-Bahy ZM. Synthesis and characterization of polyamidoxime chelating resin for adsorption of $\mathrm{Cu}(\mathrm{II}), \mathrm{Mn}(\mathrm{II})$ and $\mathrm{Ni}(\mathrm{II})$ by batch and column study. J Environ Chem Eng. 2016;4(1):276-86.

8. Rossiter GJ, Carey KC. Cooper recovery from leach liquors using continuous ion exchange.

9. Riani JC. Utilização de resinas de troca-ionica em efluentes de galvanoplastia. 2008 .

10. Harland CE. Ion Exchange Theory and Practice. 1994.

11. Zainol Z, Nicol MJ. Ion-exchange equilibria of $\mathrm{Ni2}+, \mathrm{Co} 2+, \mathrm{Mn} 2+$ and $\mathrm{Mg} 2+$ with 
iminodiacetic acid chelating resin Amberlite IRC 748. Hydrometallurgy [Internet]. Elsevier B.V.; 2009;99(3-4):175-80. Available from:

http://dx.doi.org/10.1016/j.hydromet.2009.08.004

12. Marston CR, Rodgers M. Process for separating copper and nickel from cobalt containing solutions. 2010;1(19):2010-2.

13. Zaganiaris EJ. Ion Exchange Resins and Adsorbents in Chemical Processing 2013.

14. Dow Chemical Company. Ion Exchange Resins for Chemical Processing [Internet]. [cited 2016 Apr 5]. Available from:

http://msdssearch.dow.com/PublishedLiteratureDOWCOM/dh_07c8/0901b803807c84 88.pdf?filepath=liquidseps/pdfs/noreg/177-02437.pdf\&fromPage=GetDoc

15. Bhatt RR, Shah BA. Sorption studies of heavy metal ions by salicylic acidformaldehyde-catechol terpolymeric resin: Isotherm, kinetic and thermodynamics. Arab J Chem. King Saud University; 2015;8(3):414-26.

16. Ceglowski M, Schroeder G. Preparation of porous resin with Schiff base chelating groups for removal of heavy metal ions from aqueous solutions. Chem Eng J. 2015;263:402-11.

17. Liebenberg CJ, Dorfling C, Bradshaw SM, Akdogan GA, Eksteen JJ. The recovery of copper from a pregnant sulphuric acid bioleach solution with developmental resin Dow XUS43605. J South African Inst Min Metall. 2013;113(5):389-97. 